\title{
Conflict and integration of spatial attention between disconnected hemispheres
}

\author{
S Ishiai, Y Koyama, T Furuya
}

\begin{abstract}
Objectives-To clarify how the disconnected hemispheres perceive a line and bisect it with successful or unsuccessful integration of spatial attention.

Methods-Eye movements were recorded when a patient with an extensive callosa infarction bisected horizontal lines. The lesion extended into the left cingulate gyrus.

Results-When the patient bisected lines with the right hand, the gaze was initially directed rightward and shifted further to the right side with the execution of manual response, which resulted in rightward errors. Shortly after bisection, rapid ocular searches occurred to the left side, whereas the rightward errors did not decrease throughout the trials. When using the left hand, there was no deviation of the gaze before presentation of lines. In the first few trials, the patient bisected the line with a leftward error and then searched rapidly to the right side. The subsequent bisections were almost accurate, as the subjective midpoint was placed near the point of the initial fixation that fell around the true centre. Ocular searching was mostly absent during and after line bisection.
\end{abstract}

Conclusions-In callosa disconnection, left unilateral spatial neglect may appear when use of the right hand induces a rightward bias in the attentional control of the left hemisphere and damage to its cingulate gyrus inhibits interhemispheric integration of attention. Resultant rightward errors of line bisection often cause interhemispheric conflict of attention, as the right hemisphere perceives the longer extent on the left side. By contrast, the disconnected but intact right hemisphere may bisect a line accurately by integrating attention to the extents perceived in the left and right visual fields.

(F Neurol Neurosurg Psychiatry 2001;71:472-477)

Keywords: callosal disconnection; attention; line bisection; neglect

Split brain patients exhibit various disconnection syndromes when tested specifically, whereas apparent disabilities rarely occur in the daily activities except in the early postoperative period. ${ }^{1}$ When presented with a horizontal line, the disconnected hemispheres perceive only the contralateral extent, unless eye movements bring their respective visual half field to cover the whole extent. However, patients with callosotomy show no difficulty in bisecting lines. ${ }^{23}$ By contrast, the combination of rightward bisection error with the right hand and accurate or slightly leftward bisection with the left hand has been reported after callosa infarction accompanying a lesion in the medial part of the left hemisphere. ${ }^{45}$

Recent studies ${ }^{67}$ challenged the hypothesis that split brain patients have only one integrated spatial attention. ${ }^{8} 9$ The attentional systems of the two cerebral hemispheres may work independently in the experimental conditions where the fixation is controlled. However, voluntary location based attention may be integrated interhemispherically. ${ }^{7}$ If each disconnected hemisphere distributes attention solely to its own perception of a line through the contralateral visual field, large eye movements towards the ipsilateral side would be necessary for accurate bisection. We report for the first time detailed analyses of eye movements when a patient with a callosal infarction bisected lines with either the right or left hand. We aimed to clarify how the disconnected hemispheres perceive a line and bisect it with successful or unsuccessful integration of spatial attention. We expected that integration or conflict of attentional control between the two hemispheres would appear as characteristic eye movements.

\section{Case report}

A 50 year old right handed man was examined a month after an infarction that damaged the corpus callosum. Brain MRI disclosed an extensive callosa lesion that spared only the rostrum and the inferior half of the genu (fig 1). The lesion extended into the subcortical white matter of the left cingulate gyrus. The patient exhibited most disconnection signs that would appear after surgical section of the corpus callosum. The presence of anomia and alexia in the left visual field also indicated the lesion extension to the splenium. ${ }^{10}$ His left hand sometimes behaved in conflict with the voluntary actions of the right hand. He additionally showed typical left unilateral spatial neglect when using the right hand. In the line cancellation test, the left sided 15 lines were omitted on the page where 30 lines were scattered. The patient bisected $200 \mathrm{~mm}$ lines with rightward errors of about $70 \mathrm{~mm}$ from the actual centre. When using the left hand, he cancelled all lines and bisected lines with small leftward errors, which indicated mild right neglect. Neglect was not apparent in the locomotion and the daily activities with both hands used. No apparent tendency to gaze deviation was seen in these 


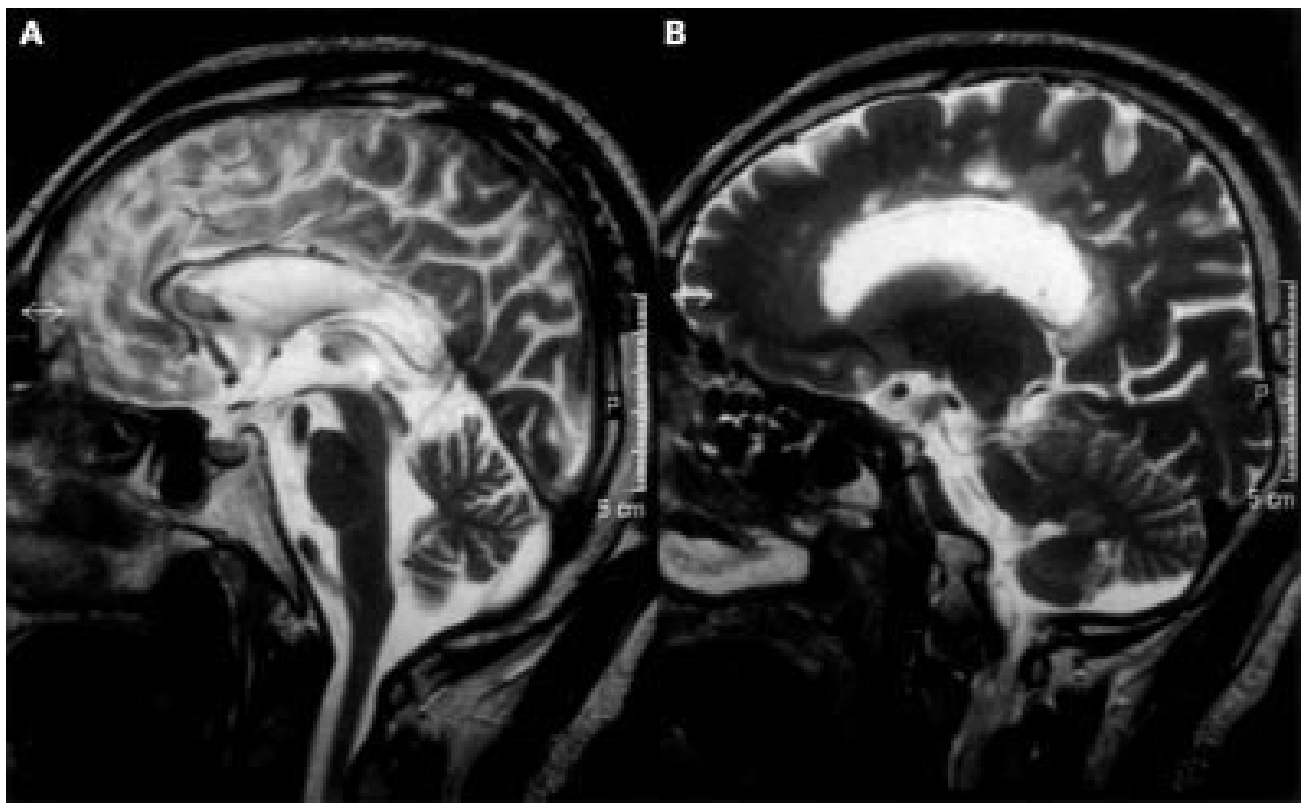

Figure 1 Sagittal T2 weighted MR images showing an extensive callosal infarction $(A)$ and the lesion extension into the subcortical white matter of the left cingulate gyrus (B).

situations and the spontaneous conditions where no specific visuospatial task was performed. After the verbal instruction by the examiner, the patient was able to move the eyes without any restriction of range. $\mathrm{He}$ gave informed consent to participate in this study, and the ethics committee of our institute approved the protocol of the experiment.

\section{Methods}

The line bisection test was performed on a 12 inch liquid crystal display monitor with a touch panel (Gunze, AV7624FT). The resolution was set at $600 \times 800$ pixels. This system was controlled by a personal computer (IBM, ThinkPad 310). The patient faced the display on the desk top with its centre positioned in the sagittal midplane of the trunk. Head movement was not restricted. The viewing distance was about $400 \mathrm{~mm}$. Before starting each bisection trial, the hand was placed naturally and comfortably in front of the display. A horizontal black line $2 \mathrm{~mm}$ wide was presented against the white background at the level of the centre of the display 1 second after a short alarm sound. The patient bisected the line by pointing to the subjective midpoint with a pencil-like pointer. The computer recorded its location to the closest millimetre and produced a short vertical mark $10 \mathrm{~mm}$ tall and $1 \mathrm{~mm}$ wide at that point. The line with the mark was displayed for 2 seconds after the bisection. No feedback was given to the patient about the error.

We used 150 and $200 \mathrm{~mm}$ lines, for which patients with neglect after right hemispheric lesions often show rightward errors of bisection. The patient underwent four blocks of line bisection trials. Each block contained eight trials: four trials were requested for either line length with the order of presentation randomised. To balance practice and fatigue effects between the hands used, the patient bisected the lines with the right hand in the first and fourth blocks and with the left hand in the second and third blocks. He thereby performed eight trials for each length with either the right or left hand.

We recorded the eye fixation pattern and the process of line bisection with a system involving an eye camera (EMR-7, NAC Inc). The patient could perform the task under natural conditions. The head unit of this apparatus, which includes the eye camera and a scene camera, weighs $350 \mathrm{~g}$ and is connected to the control unit by flexible cables. The corneal reflection detected by the eye camera is transduced electronically into the eye mark and then superimposed on the picture from the scene camera. The movements of the eye mark were adjusted so that when the patient looked at known points in the visual scene, the mark lay over these points in the combined eye-scene picture. Calibration was accomplished without difficulty, as stable fixation was obtained at the centre of the monitor and the points on either side. The eye-scene picture was recorded with a video recorder at a speed of 30 frames/ second. The location of the patient's fixation almost always moved on the line during line bisection. We therefore analysed lateral movements of the fixation relative to the presented line.

\section{Results}

Deviations of the subjective midpoint were measured from the true centre and given positive values when they were directed to the right side. The patient consistently erred rightward with the right hand. The mean deviation was 54.1 (SD 6.4) $\mathrm{mm}$ for the lines $150 \mathrm{~mm}$ long and 70.1 (4.4) $\mathrm{mm}$ for the lines $200 \mathrm{~mm}$ long. Figure 2 A shows a representative eye fixation pattern for the bisection of a $200 \mathrm{~mm}$ line. When the patient prepared to start the trial, his gaze was directed to the right side position on 

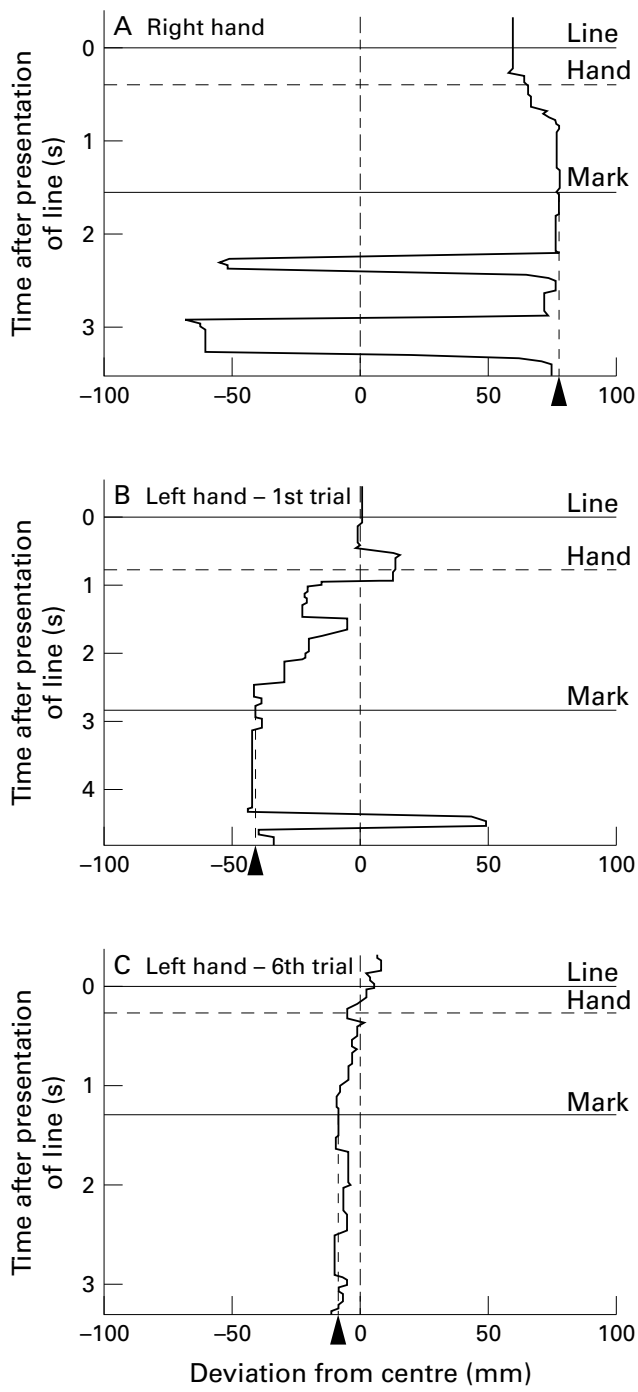

Figure 2 Eye fixation patterns in line bisection. (A) Representative trial with the right hand. (B) First trial with the left hand. (C) Sixth trial with the left hand. The line length was $200 \mathrm{~mm}$. "Line", "hand", and "mark" indicate the time of line presentation, the time when the hand started to move, and the time when the subjective midpoint was marked, respectively. The eye fixation patterns are shown from the period of 0.33 seconds before presentation of the line. The arrowhead beneath the horizontal axis shows the location of the subjective midpoint.

the blank display. After presentation of the line, rightward drifts occurred with the execution of the manual response, which brought the fixation to the point where the subjective midpoint was later placed. The mean duration from presentation of a line to bisection was 1.81 (SD 0.60) seconds. During this period, no leftward ocular search was found. By contrast, soon after pointing to the subjective midpoint (mean 1.05 (SD 0.25) s), one or two rapid leftward searches occurred in all but one of the 16 trials (fig 3A). The duration of these searches was very short (mean 0.29 (SD 0.04) s) and the gaze quickly returned to the rightward position. The line bisection performance with the right hand was unchanged throughout the trials. The eye fixation pattern showed that before placement of the mark, the patient's left hemisphere perceived the line extent between the location of the initial fixation and the right end point through the right visual field. The subjective midpoint divided the perceived extent into two segments, although it was placed near the point of the initial fixation in a few exceptional trials (fig 3A). The best fitting regression for the distance of the right segment was $7.86+0.40 \times$ perceived extent $\left(r^{2}=0.76\right)$.

When the patient bisected the lines with the left hand, the initial points of fixation were not significantly deviated from the true centre (mean deviation $-4.2 \mathrm{~mm} ; t=-1.65$ ) and located more leftward compared with those in the bisections with the right hand $(t=11.22$, $\mathrm{p}<0.001$, fig $3 \mathrm{~B}$ ). The mean duration from presentation of a line to bisection was 1.52 (SD 0.69 ) seconds. In the first four trials, the gaze moved leftward after presentation of the line, with saccadic movements interspersed (fig 2 B). This shift of fixation accompanied the movement of the left hand, and the subjective midpoint was displaced to the left side. After placement of the subjective midpoint, rapid rightward searches occurred in three of the four trials (figs $2 \mathrm{~B}$ and $3 \mathrm{~B}$ ). In the remaining 12 trials, however, the mean deviation was -3.3 (SD 5.6) $\mathrm{mm}$ for the $150 \mathrm{~mm}$ lines and -6.6 (SD 11.8) $\mathrm{mm}$ for the $200 \mathrm{~mm}$ lines. The subjective midpoint was placed near the point of the initial fixation, which fell around the true centre of the line (fig $2 \mathrm{C}$ ). No apparent search was made to either side during line bisection, and in only one trial, a rightward search occurred after pointing to the subjective midpoint (fig $3 \mathrm{~B} ; 150 \mathrm{~mm}$, trial 6). In an exceptional trial, the fixation was initially deviated to the left side, whereas its gradual shift toward the centre resulted in the nearly accurate bisection (fig $3 \mathrm{~B} ; 150 \mathrm{~mm}$, trial 4).

\section{Discussion}

In the bisection of horizontal lines, the patient with a callosal infarction showed rightward errors with the right hand. The gaze was shifted to the right side when he prepared to start the bisection trials. After presentation of the lines, additional rightward drifts of the fixation occurred with the hand movement to point to the subjective midpoint. Patients with neglect after right hemispheric lesions were reported to show a rightward shift of the median fixation when they examined an entire line before touching its centre. ${ }^{11}$ The gaze shift was, however, less obvious in the free viewing of a line with no instruction to bisect it. ${ }^{12}$ The patient of the present study showed no apparent bias of eye movements when he did not use the right hand to perform visuospatial tasks.

According to the premotor theory of Rizzolatti and Gallese, ${ }^{13}$ spatial attention is a correlate of the organisation of motor acts. Patients with unilateral spatial neglect after right hemispheric lesions sometimes show more pronounced left neglect with the right hand than with the left hand. ${ }^{14}{ }^{15}$ Use of the right hand may activate the sensorimotor circuits in the left hemisphere and enhance the rightward bias of attention, although some complicated interactions have been reported between the hand used and the space of motor 

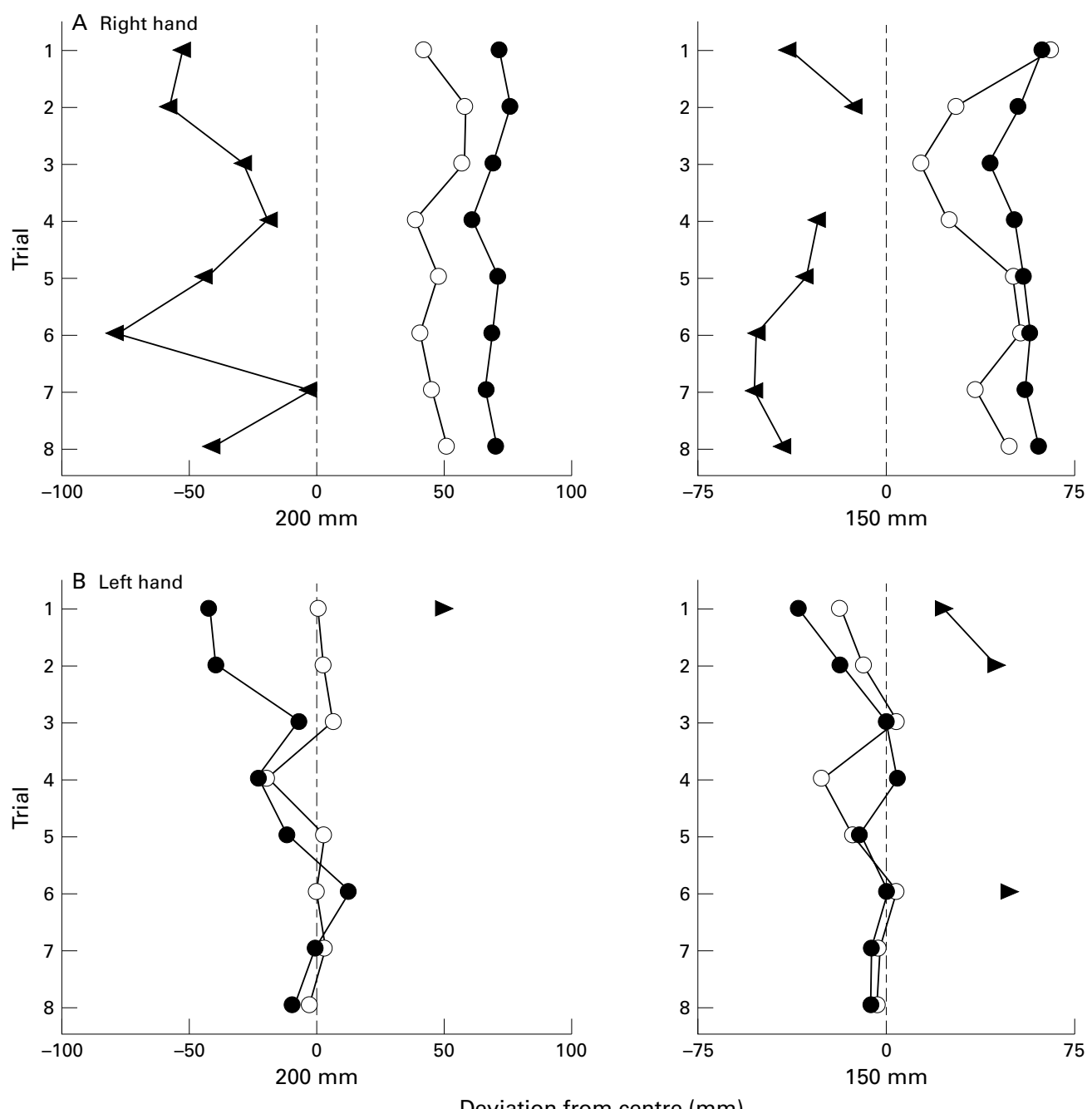

Deviation from centre $(\mathrm{mm})$

Figure 3 Relation between fixation point and subjective midpoint. Open circles, initial points of fixation on line; closed circles, locations of subjective midpoint. (A) Bisections with the right hand. In most trials, leftward searches occurred after bisection, and their extreme points are indicated as leftward triangles. (B) Bisections with the left hand. Rightward triangles show the extreme points of rightward searches that occurred after four bisection trials.

execution. ${ }^{16}{ }^{17}$ Our patient placed the hand near the body midline before starting the bisection trials. Also, the response required minimal movements of the proximal arm, which might be controlled partly by the ipsilateral hemisphere. ${ }^{9}$ We therefore consider that the preparation and execution of response with the right hand may have activated the left hemisphere to bias its own attentional control towards right space. In patients with callosotomy, such activation would not cause pathological rightward shifts of the gaze or the response. Surgical split brain patients showed no apparent left neglect or rightward error when using the right hand in the tactile rod bisection task. ${ }^{2}$ In the free viewing bisection of lines, a patient with complete commissurotomy showed a slight leftward bias using either right or left hand to press the response key. ${ }^{3}$ The lesion of our patient, however, extended into the left cingulate gyrus, which is a component of the neural network for directed attention. ${ }^{18}$ Medial frontal lesions accompanying callosa disconnection were considered to prevent extracallosal transmission of attentional information..$^{5}$ In callosa disconnection, the gaze may be shifted to the right side, when use of the right hand induces a rightward bias in the attentional control of the left hemisphere and damage to its cingulate gyrus inhibits interhemispheric integration of attention. This gaze shift seems to be the primary cause of rightward errors or left neglect in line bisection.

In split brain patients, crude cross integration of the visual fields may be achieved by interactions between cortical and subcortical visual areas. ${ }^{19}$ Limited perceptual information may be transferred also via the spared callosal fibres after incomplete section of the corpus callosum. ${ }^{20}$ When using the right hand, our patient bisected the line extent perceived within the right visual field, which indicated the absence of perceptual integration between the two hemispheres. We therefore consider that the patient showed left neglect according to the intrahemispheric attentional and perceptual processing of the left hemisphere (fig $4 \mathrm{~A}$, left). There are controversies as to whether the intact left hemisphere is able to distribute attention to either side of space ${ }^{21}$ or only to right space. ${ }^{518}$ Some dysfunction of the left hemisphere has been suggested to contribute to manifestation 
A Left hemisphere - right hand

During LB
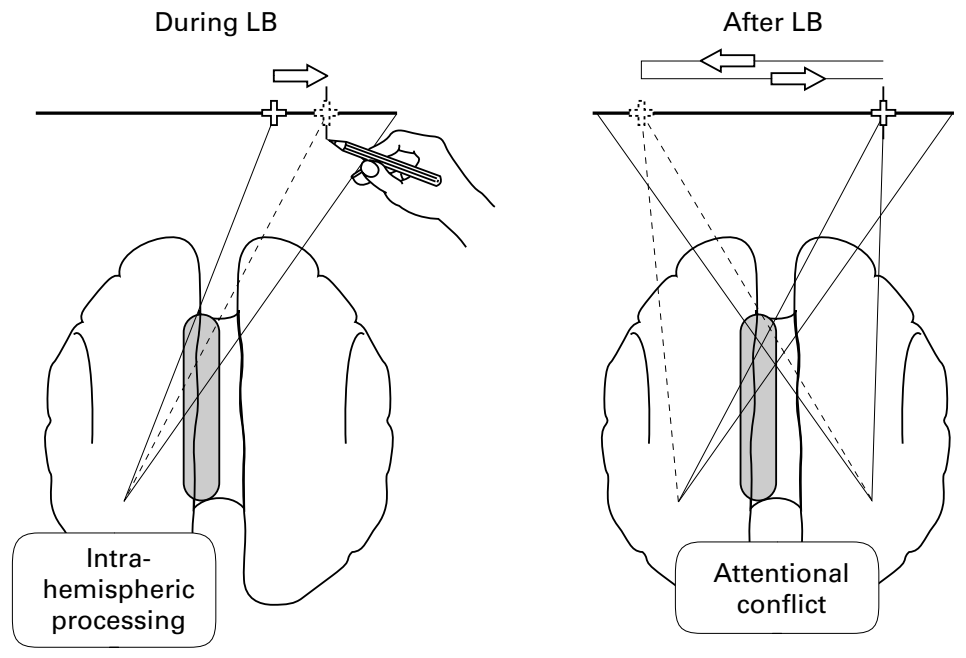

B Right hemisphere - left hand

During and after LB

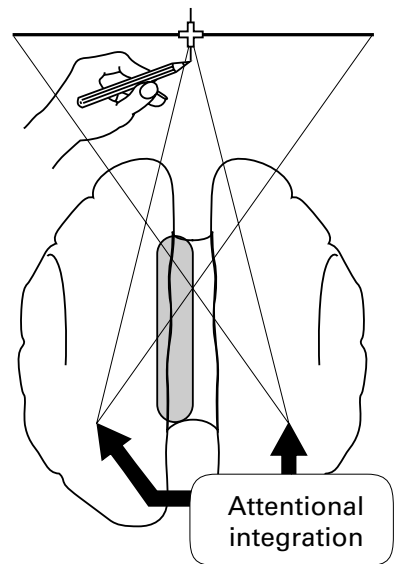

Figure 4 (A) Line bisection (LB) with the right hand. (B) Line bisection with the left hand for nearly accurate trials.

of neglect after right hemispheric lesions. ${ }^{2}{ }^{21} \mathrm{It}$ is possible that the rightward shift of the gaze was enhanced because the attentional network of the left hemisphere had a pathological contralateral bias due to the damage to the cingulate component.

The errors of bisection induced conflicts in the control of spatial attention between the two hemispheres. When bisecting with the left hand, such conflict disappeared after the errors were corrected in the first few trials. By contrast, the rightward errors with the right hand consistently induced interhemispheric conflicts of attention (fig $4 \mathrm{~A}$, right). The patient's right hemisphere saw the longer left extent through the left visual field while the left hemisphere or the right hand was bisecting the lines. After bisection, the right hemisphere's attentional system, which had been subcortically suppressed to control eye movements, produced overt leftward shifts of attention. When the fixation was moved near to the left end point, the left hemisphere may have appreciated its own rightward errors of bisection. ${ }^{21}$ The repetitive rightward errors with the right hand may reflect the subcortical lesion extension into the left cingulate gyrus. Recent studies suggested a specific role of the anterior cingulate cortex to monitor competition between incompatible processes or responses during task performance. ${ }^{22}{ }^{23}$ This monitoring may be responsible mainly for motor responses. ${ }^{24}$ The left hemisphere with the anterior cingulate dysfunction may have failed to modulate the rightward bias of attention even after detecting the rightward errors of bisection. However, patients with left neglect after right hemispheric lesions also show rightward bisection errors repetitively after feedback of their performance. $^{25}$ Their lesions often spare the anterior cingulate cortex. When our patient bisected lines with the right hand, some functional specialisation or impairment of the left hemisphere outside the cingulate gyrus might also contribute to the consistent appearance of neglect.

When using the left hand, the patient gazed at the point near the centre of the display before the lines were presented. In the first four trials, the gaze was shifted to the left side when he started to point to the subjective midpoint. As in the bisections with the right hand, execution of the response with the left hand may have activated the right hemisphere to bias its attentional control toward left space. The contralateral left hemisphere perceived the longer right extent of the line when the gaze was directed to the left side. After bisection, this perception probably induced rightward searches, in which the left visual field covered the line extent enough to appreciate the leftward displacement of the mark (fig $2 \mathrm{~B}$, last rightward search). The intact right anterior cingulate cortex ${ }^{22} 23$ probably modulated the attentional control of the right hemisphere to prevent leftward gaze shifts in the ensuing trials, where the bisections were almost accurate. When the initial fixation fell on the leftward point in an exceptional trial, the patient shifted the gaze to the centre and bisected it accurately. Accordingly, his nearly accurate bisection with the left hand required minimal movements of fixation on the midportion of the lines. Besides, ocular searching was mostly absent after bisection. Some normal subjects bisect lines similarly without apparent searches to either side. ${ }^{21}$ The disconnected hemispheres perceive only the contralateral extent of a line, unless their respective visual half field cover the whole extent with the eyes moved. The disconnected but intact right hemisphere may integrate attention to the perceptions from the left and right visual fields (fig $4 \mathrm{~B}) .^{26}$ Most split brain patients bisect lines normally with either hand. Studies on eye movements in line bisection should be applied to surgical cases to determine if the disconnected but intact "left" hemisphere also has an integrative ability of spatial attention.

This work is supported by a Grant in Aid for Scientific Research (C) to Sumio Ishiai from the Ministry of Education, Science, Sports, and Culture, Japan. 1 Sperry RW, Gazzaniga MS, Bogen JE. Interhemispheric
relationships: the neocortical commissures; syndromes of
hemisphere disconnection. In: Vinken PJ, Bruyn GW, eds. 
Handbook of clinical neurology. Vol 4. Amsterdam: NorthHolland, 1969:273-90.

2 Plourde G, Sperry RW. Left hemisphere involvement in left spatial neglect from right-sided lesions: a commissurotomy study. Brain

3 Corballis MC. Line bisection in a man with complete forebrain commissurotomy. Neuropsychology 1995;9:147-56.

4 Goldenberg G. Neglect in a patient with partial callosal disconnection. Neuropsychologia 1986;24:397-403.

5 Kashiwagi A, Kashiwagi T, Nishikawa T, et al. Hemispatial neglect in a patient with callosal infarction. Brain 1990;113:1005-23.

6 Luck SJ, Hillyard SA, Mangun GR, et al. Independent hemispheric attentional systems mediate visual search in split-brain patients. Nature 1989;342:543-5.

7 Corballis MC. Visual integration in the split brain. Neuropsychologia 1995;33:937-59.

8 Gazzaniga MS. Perceptual and attentional processes following callosal section in humans. Neuropsychologia 1987;25: ing callo $119-33$.

9 Gazzaniga MS. Principles of human brain organization derived from split-brain studies. Neuron 1995;14:217-28.

10 Gazzaniga MS, Freedman H. Observations on visual processes after posterior callosal section. Neurology 1973 23:1126-30

11 Barton JJS, Behrmann M, Black S. Ocular search during line bisection: the effects of hemi-neglect and hemianopia. Brain 1998;121:1117-31.

12 Ishiai S, Furukawa T, Tsukagoshi $\mathrm{H}$. Eye-fixation patterns in homonymous hemianopia and unilateral spatial neglect. Neuropsychologia 1987;25:675-9.

13 Rizzolatti G, Gallese V. Mechanisms and theories of spatial neglect. In: Boller F, Grafman J, eds. Handbook of neuropsychology. Vol 1. Amsterdam: Elsevier, 1988:223-46.

14 Joanette Y, Brouchon M, Gauthier L, et al. Pointing with left vs right hand in left visual field neglect. Neuropsychologia 1986;24:391-6.
15 Halligan PW, Marshall JC. Laterality of motor response in visuo-spatial neglect: a case study. Neuropsychologia 1989;

16 Halligan PW, Marshall JC. Perceptual cueing and perceptuo-motor compatibility in visuo-spatial neglect: a single case study. Cogn Neuropsychol 1989;6:423-5.

17 Robertson IH, North N. Spatio-motor cueing in unilateral eft neglect: the role of hemispace, hand and motor activation. Neuropsychologia 1992;30:553-63.

8 Mesulam M-M. Large-scale neurocognitive networks and distributed processing for attention, language, and memory. Ann Neurol 1990;28:597-613.

19 Holtzman JD. Interactions between cortical and subcortical visual areas: evidence from human commissurotomy patients. Vision Res 1984;24:801-13.

20 Funnell MG, Corballis PM, Gazzaniga MS. Cortical and subcortical interhemispheric interactions following partial and complete callosotomy. Arch Neurol 2000;57:185-9.

21 Ishiai S, Furukawa T, Tsukagoshi H. Visuospatial processe of line bisection and the mechanisms underlying unilateral spatial neglect. Brain 1989;112:1485-502.

22 Carter CS, Braver TS, Barch DM, et al. Anterior cingulate cortex, error detection, and the online monitoring of

23 Barch DM, Braver TS, Sabb FW, et al. Anterior cingulate and the monitoring of response conflict: evidence from an fMRI study of overt verb generation. $\mathcal{F} \operatorname{Cog} n$ Neurosci 2000 ; 12:298-309.

24 Turken AU, Swick D. Response selection in the human anterior cingulate cortex. Nat Neurosci 1999;2:920-4.

25 Seki K, Ishiai S, Koyama Y, et al. Unassociated responses to two related task demands: a negative factor for improvement of unilateral spatial neglect. Neuropsychologia 1999; 37:75-82.

26 Holtzman JD, Sidtis JJ, Volpe BT, et al. Dissociation of spatial information for stimulus localization and the control of attention. Brain 1981;104:861-72. 